\title{
On the Verification of State-Coding in STGs
}

\author{
Kuan-Jen Lin and Chen-Shang Lin \\ Department of Electrical Engineering \\ National Taiwan University
}

\begin{abstract}
One primary property for an Signal Transition Graph (STG) to be synthesized in an asynchronous circuit is the realizable state coding (RSC) - more relaxed than USC. A procedure and its theoretical basis have been develope$d$ to verify the RSC property of a STG which contains multi-cycle signals and has a underlying free-choice Petri net. The procedure is carried out wholly on STG domain and is successful to verify the RSC of benchmarks[3].
\end{abstract}

\section{Introduction}

The synthesis of asynchronous circuits from specifications given in Signal Transition Graphs (STGs) has become an active research area $[2,4,5,6,7]$ since its first introduction by $\mathrm{Chu}[1]$. One major factor of its popularity is the conciseness of the representation, the complexity of which is only linearly proportional to the number of signals involved in the specification.

The prevalent synthesis procedure from STG descriptions is comprised of (1) ensuring that the STG is feasible for realization; (2) synthesizing hazard-free circuits. An STG should possess some properties before the circuit can be realized. One primary requirement is to ensure the corresponding state-diagram of STG to have a realizable state-coding. Usually the more restricted unique state coding property is sought. The verification and rectification for realizable state-coding are non-trivial tasks, since the size of state diagram is exponential with respect to the signal number. Furthermore, the design optimizations are much intended to be explored on higher level description. Therefore, it is much desirable to suggest methods by which synthesis tools or designers can satisfy this requirement directly on STG domain rather than state diagram.

Previous works $[4,5]$ had presents techniques based on lock-relations among signals to treat the state-coding problem wholly on STG domain. However, their STG descriptions are restricted to be marked graphs which exclude any conditional behaviors. Furthermore, the more constrained unique state-coding are considered in their works, which might incur extra cost.

In this paper, new theoretical foundations and a new verification procedure based on transitive lock relation[5] are developed to solve state-coding problem wholly on STG domain for STGs containing multi-cycle signals and con- ditional behaviors. The state coding problem considered here is the realizable state-coding which is a more relaxed requirement than the unique state coding. For STGs with multi-cycle signals, single-cycle transformation is proposed to transform the problem in to ones with single-cycle signals. For STGs with conditional behaviors, two novel operation, branch reversing and self-loop unfolding are proposed to significantly simplify the verification process. The proposed algorithmic approaches have been shown successful on over thirty examples from academic and industry.

In next section, the state coding problem and relevant established results will be reviewed. Then the verification of state coding for STG with multi-cycle signals will be presented. In Section 4, two novel operations, branch reversing and self-loop unfolding, will be introduced to facilitate the solving state-coding problem on STG with conditional behaviors. A procedure incorporating these operations will then be illustrated with an example. Finally, we will give our evaluation results on over thirty examples and then the conclusion.

\section{STG Synthesis and State- Coding}

An STG can be viewed as an interpreted Petri net. The STG under consideration has the underlying Petri net being a free-choice net. In such STG, the places of single fanin and single fanout of the underlying net are removed and only two types of nodes are retained: the signal transitions and the multiple fanin or multiple fanout places. The transitions of signal $a$ denoted by $a+$ and $a-$ represent the rising and falling transitions respectively, and if it has underline, it is an input transition. A transition of signal $a, t_{a}$, can be either $a+$ or $a-$ and if $t_{a}=a+$ then $\overline{t_{a}}=a-$. A signal is single-cycle if it only contains two transitions, otherwise it is multi-cycle. In STG, if there is an arc from a transition $t_{1}$ to another transition $t_{2}$, we denote it by $t_{1} \rightarrow t_{2}$. And $t_{1} \Rightarrow t_{2} \ldots \Rightarrow t_{k}, t_{1}, t_{2}, \ldots t_{k}$ are in a loop and these transitions occur in the order of their indexes.

A live and safe free-choice Petri-net can be covered by a set of live and safe MG-components and SMcomponents[1]. An Marked-Graph(MG) is a net in which each place has at most one fanin and at most one fanout. 
By duality, an State-Machine(SM) is a net in which each transition has at most one fanin and at most one fanout. The MG can model concurrent behaviors and SM conditional behaviors.

For a given STG to be synthesized, it should possess certain properties as follows[1].

Definition (Liveness) A STG is live iff

(1) the underlying Petri net is live and safe,

(2) every SM-component contains exactly one token,

(3) for each signal $a$, transitions $t_{a}$ and $\overline{t_{a}}$ are ordered. Definition:(Realizable State Coding(RSC))

A live STG has RSC property iff any two states which enable different sets of non-input signal transitions have distinct state codes.

Note that the RSC is more relaxed than the USC (Unique State Coding) which requires all state codes to be distinct. To satisfy RSC property before circuit realization is a mandatory task. However, it is a non-trivial task, since the size of state diagram is exponential with respec$t$ to the signal number. Here the verification of RSC is to be approached on the STG domain. The complexity of the problem depends on the underlying Petri net of the given STG. We denote an STG by STG/MG if it only contains exactly one marked graph and by STG/FC if it has input free-choice behaviors (conditional behaviors). An STG/MG is an STG/MG-S, if each signal is single-cycle.

In an STG/MG-S, special relations between signals have been shown to have significant impact on the realizability. Definition (Lock)

In an STG/MG, there is a lock relation between singlecycle signals $a$ and $b$, denoted as $a L b$, iff

$a+\Rightarrow b+\Rightarrow a-\Rightarrow b-$

$\vee a+\Rightarrow b-\Rightarrow a-\Rightarrow b+$

Definition (Transitive Lock)

In an STG/MG, there is a transitive lock relation between single-cycle signals $a$ and $b$, denoted as $a L^{t} b$, iff

$a L b \vee\left(a L^{t} c \wedge c L^{t} b\right), c$ is another signal in STG.

Note that the lock relation is not defined for a multicycle signal. The transitive lock relation is crucial not only for state-coding problem but also the synthesis of hazardfree implementations[8]. The following useful theorem is derived in $[4,5]$ as a sufficient condition to satisfy RSC.

Theorem 1: A live STG/MS-S has USC(Unique State Coding) property if there is $L^{t}$ relation between any two signals.

\section{RSC for STG/MG}

For a live STG/MG, our basic strategy is to transform or split each cycle of a multi-cycle signal into a distinct singlecycle signal and then to apply the results for STG/MG-S to solve the realizable state coding problem. The detail will be described in this section.

Let us firstly describe the more general strategy. Let $x_{1}+, x_{1}-, x_{2}+, \ldots, x_{n}+, x_{n}-$ be all transitions of $x$ and with the relation: $x_{1}+\Rightarrow x_{1}-\Rightarrow x_{2}+\Rightarrow$ $x_{2}-\Rightarrow \ldots x_{n}+\Rightarrow x_{n}-$. Since there are two ways to group adjacent transitions into a cycle, the corresponding two single-cycle transformations are as follows: (1) odd order: $\left(x_{1}+, x_{1}-\right),\left(x_{2}+, x_{2}-\right), \ldots,\left(x_{n}+, x_{n}-\right) ;(2)$ even order: $\left(x_{1}-, x_{2}+\right),\left(x_{2}-, x_{3}+\right), \ldots,\left(x_{n}-, x_{1}+\right)$.

Based on the transformation strategy, the following theorem allows us to verify RSC property for STG/MG with multi-cycle signals directly on STG domain.

Theorem 2: A live STG/MG has realizable state coding if in each single-cycle transformation on all multi-cycle signals, $a L^{t} b$ is true for each non-input signal $a$ and any of it's enabling signal $b$.

Proof: see [11].

The usefulness of the above results evidently depend$s$ on the number of multi-cycle signals in the STG, since it incurs $\left(2^{N}\right)$ possible transformation combinations for $N$ multi-cycle signals. To reduce the complexity, we have observed that for a multi-cycle signal satisfying certain conditions, it can be considered as a set of independent singlecycle signals when checking RSC property by Theorem 2 . In other words, only either odd- or even-order transformation of the signal is sufficient to derive RSC. This desirable condition is described in the following.

Virtual Single-Cycle Signal: A signal $m$ with $n$ cycle can be considered as a set of single-cycle signals from it's even(odd) order transformation if

$\forall i, i=0,2, \ldots, 2 n-2(i=1, \ldots, 2 n-1)$, there are two real or virtual single-cycle signal transitions $t_{x 1}$ and $t_{x 2}$ having $t_{x 1}=\overline{t_{x 2}}$ or $x 1 L^{t} x 2$ relation, such that $t_{m}^{i} \Rightarrow t_{x 1} \Rightarrow$ $t_{m}^{i+1} \Rightarrow t_{m}^{i+2} \Rightarrow t_{x 2} \Rightarrow t_{m}^{i+3}$, where $t_{m}^{k}=t_{m}^{k \bmod 2 n}$.

The application and importance of this technique are shown by the following theorem.

Theorem 3: For a live STG/MG, after virtual singlecycle signal transformation, if it satisfies the RSC condition of Theorem 2, the original STG/MG also have RSC property.

Proof: see [11].

To illustrate the approach, we take the example shown in Fig. 1, where the /number is used to distinguish between multiple transitions of the same signal. In the beginning the signal $c$ is found to be virtual single-cycle signals by $e$. Then $e, d, c 1$, and $c 2$ are found to have $L^{t}$ relation between each other. By their transitions, $e+$ and $c-/ 2, b$ thereby can be regarded as two virtual single-cycle signals. Fig. 1(c) shown the final lock-graph. The example is found to have RSC property but not USC.

With a preprocessed Relation-Table[10], if all multicycle signals can be iteratively treated as virtual singlecycle signals, the complexity of verification will be $O\left(N^{2} \times\right.$ $\left.M+M^{2} \times N\right)$, where $N$ is the number of single-cycle signals and $M$ of multi-cycle. Specifically, for one iteration, in which we try to transform all remaining multi-cycle signals using real or transformed virtual single-cycle signals, we need $\left(N^{2}\right)$ for the derivation of $L^{t}$ relation and $(N \times M)$ for virtual single-cycle signal transformations. In worst case, $M$ iterations are required.

The virtual single-cycle concept has been found to be extremely useful in practical cases. In our experiment, most of STG/MGs derived from Berkely[3] can be evaluated by 


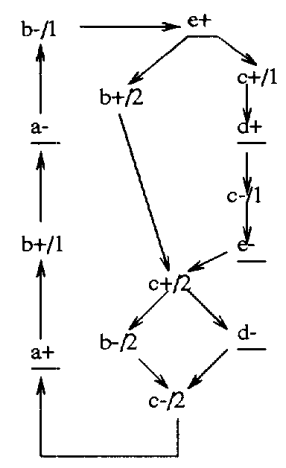

(a)

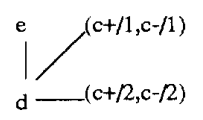

(b)

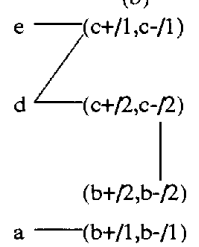

(c)
Figure 1: (a) An initial STG with two multi-cycle signals and (b) it's lock graph after $c$ transformed to virtual single-cycle signals and (c) it's lock graph after $c$ and $b$ transformed to virtual single-cycle signals.

this technique only.

\section{Branch Reversing and Self- loop Unfolding}

Since a live STG/FC can be covered by a set of live STG/MGs[1], it is possible to verify the RSC of the STG/FC from those of MG components and their combinations. Although it is not difficult to verify whether these smaller covering STG/MG components to have RSC based on the results in the previous section, it is not trivial to verify markings in different MG-components whether to satisfy RSC requirement. To simplify the latter task, two operations, branch reversing and self-loop unfolding, will be introduced in this section. By appropriate usage of these two operations, the STG/FC can be transformed into such a way that the RSC is checked for each covering MG component and each pair.

Consider a free-choice Petri net with a given initial marking. Define a place with multiple fanouts as a fork and a place with multiple fanins as a join. A place can be both a fork and a join. Between a fork and a set of concurrent joins, the arcs and places which form a connected segment of a covering MG-component is a branch. A branch is a self-loop if its two ends are the same place. A branch is said to be reversed if the direction of all arcs connected to transitions as well as the transition directions in the branch are reversed. In Fig. 2(b), when the branch $e-\rightarrow b+\rightarrow c+$ is reversed, the resultant net is shown in Fig. 2(c). And self-loop is said to be unfolded if its common place is split into two places such that one consists only of the fanouts to the self-loop and the original fanins except those from the self-loop, and the other place consists of the remaining fanins and fanouts. The other structural relations are retained. In other words, the self- loop is straightened as can be seen from Fig. 2(b) in which one self-loop in Fig. 2(a) is unfolded.

The application of these two operations on a live STG will still retain the liveness property. From [9], when an live and safe net is entirely reversed, it will behave as backward firings as the original. Hence, it is still live and safe, and can generate equivalent markings(one-to-one corresponding) as the original. In our application, only some specific parts of net are reversed. Those parts, in essence, can be removed and the original net is still live and safe. Based on this fact, we can prove that the liveness and safeness of net, the liveness of STG are retained after an STG/FC reversing an Branch[11]. To unfold an self-loop, can be considered as to force the self-loop to be chosen each time when the fork of self-loop is marked. In essence, they are legal firing sequences in original net. Therefore, liveness and safeness of net, the liveness of STG are retained after one self-loop is unfolded[11].

Given an initial marking, we are able to traverse the STG from this given marking and identify all the feedback branches similar to identify all feedback loops from a sequential circuit. Reversing all feedback branches except self-loops without the initial marking and unfolding those self-loops, the resultant net has the desirable property that all covering MG-components of the transformed net contain the given initial marking. In other words, the running MG contains all the tokens and thus determines the states. As a result, to verify the RSC property of the transformed net, we need only to check the RSC of each MG-component and whether two markings of different MGs have the same state code. The key question is whether the transformed net has the equivalent RSC problem as the original one. The answer is affirmative as established in the following two theorems.

Theorem 4: For a live STG/FC with initial marking $m_{0}$, after one of it's branch is reversed, each marking in original STG has a corresponding marking in the transformed STG and they have the same coding.

Proof: see [11].

From the above theorem, it can be easily shown that the USC is preserved after branch reversing. RSC can also be easily treated by observing that the predecessor and successor relation is reversed in the reversed branch.

Theorem 5: For a live STG/FC with initial marking $m_{0}$, after one of it's self-loop is unfolded, the STG has equivalent RSC problem as the original.

Proof: see [11].

\section{An RSC Verification Proce- dure for STG/FC}

Based on the discussion in the last section, the principle of RSC property verification for an STG/FC can be described.

From the previous section, given an initial marking of an STG/FC, we are able to transform the given net into a net without internal feedback branch so that each covering 


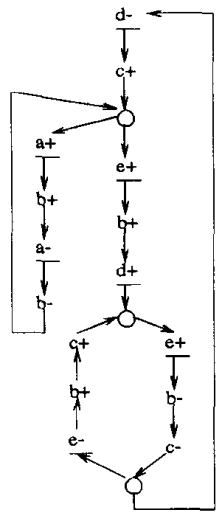

(a)

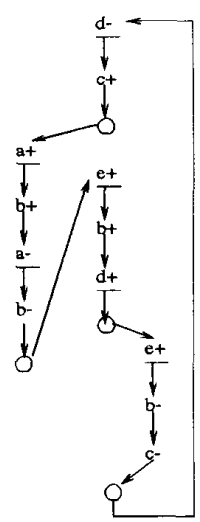

(d)

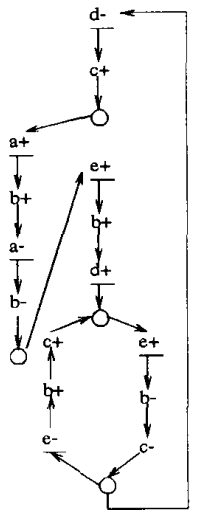

(b)

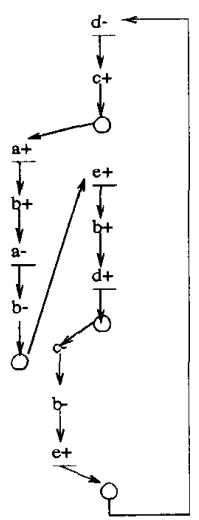

(e)

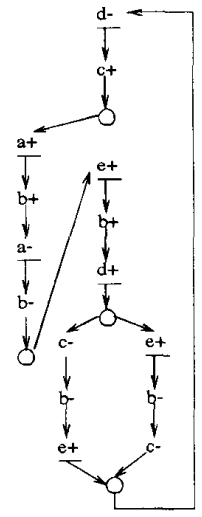

(c)<smiles>[C]1OCO[Te]CO[Te]1</smiles>

(f)<smiles>[C+]1[C+]O[Te]O[Te]OC1</smiles>

Figure 2: (a) An initial STG/FC; (b) The STG/FC from (a) unfolding one self-loop; (c) The STG/FC from (b) reversing one feedback branch; (d) (e) MGcomponents from (c); (f) Disjoint set of (d) and (e); (g) An STG/MG from (f) reversing one branch.

MG-component contains the initial marking. Hence each running MG contains all the tokens and thus determines the states. As a result, to verify the RSC property, we need only to check the RSC of each MG-component and whether two markings of different MGs have the same state code. The former can be solved by those described in Section 3 , and the latter is simplified because all MG-components have one common marking. The disjoint set between any two MG-components then contains only pairs of conflic$t$ branches which are two branches with the same fork and join. Only these conflict pairs need to be checked for state code equivalence, and each pair of the conflic$t$ branches, by branch reserving and self-loop unfolding, can be transformed into MGs with equivalent state coding problem. Since the conflict-branch pair has the same fork and join, if fork and join are distinct nodes, we can

reverse one branch of the pair such that the two connected segments of MG-components are strongly connected to become one live and safe MG. If fork and join are the same node, this means these two branches are actually two entire MG-components. One branch is then unfolded and linked to the other to become one larger MG. Both two cases allow us apply the techniques for STG/MG to verify RSC.

Based on the above descriptions, a procedure to verify the RSC property of STG/FC is given below.

Procedure: $R S C$ Verification for $S T G / F C$ :

(Given a live $S T G / F C$ with an initial marking $m_{0}$ )

1. Unfold all Self-loops not marked in $m_{0}$.

2. Reverse all Feedback-Branches.

3. Derive all transition relations.

4. Foreach $M G$-component, perform verification

5. Foreach pair of Conflict-Branch $\left(C B_{i}, C B_{j}\right)$ Reverse or Unfold $C B_{i} \Rightarrow C B_{i}^{\prime}$.

\}endforeach perform verification for $C B_{j}+C B_{i}^{\prime}$.

Let us illustrate the Procedure by an example shown in Fig. 2(a). After the first two steps, the initial STG/FC is transformed to the STG in Fig. 2(c). This transformed STG/FC has two MG-components, as shown in Fig. 2(d) and Fig. 2(e). Both these MG-components can be easily verified by techniques for STG/MG. There is one pair of conflict branch in the transformed STG, as shown in Fig. $2(f)$. We can reverse one branch to make them to be an STG/MG shown in Fig. 2(g). Again, it is verified by the method for STG/MG. The complexity of this procedure mainly depends on the number of MG-components and pairs of conflict-branches.

\section{Discussion and Conclusion}

The proposed verification procedures in this paper have been successfully evaluated with the set of STG benchmarks from Berkely[3]. Table 1 contains those STGs having multi-cycle signals or conditional behaviors. The second column shows the total number of signal transitions in STG. STG/MG and STG/FC can be distinguished by the No. of $M G$-components $(M G)$ since an STG/MG have only one MG-component. For an STG/FC, Pairs of Conflictbranches $(C B)$ is the number of STG/MGs to be processed in step 4 of the RSC Procedure to verify markings in different covering MGs. The No. of $M G$-components plus Pairs of Conflict-branches are the total times required to employ techniques for STG/MG to verify RSC. In all examples, the number is quite small and the number of transitions in each such MG, in general, is much smaller than the number in whole STG/FC.

Another useful indicator of the complexity is the number of Iterations of Virtual Single-Cycle Transforma$\operatorname{tion}(V S C)$. It is the iterations needed to transform an 
STG/MG to STG/MG-S with the virtual single-cycle signal technique. Most examples can be completed in one iteration and the complexity of RSC verification for those STG/MG is close to $\left(N^{2}\right)$, where $N$ is the number of signals in that STG. Furthermore, all cases except Lavagno.g and pe-send-ifc.g, including all STG/MGs during the RSC verification of STG/FCs, can be verified with this technique only. This demonstrates the effectiveness of virtual single-cycle signal transformation.

In this paper, we have proposed a new verification procedure and the theoretical foundations based on transitive lock relation to solve state-coding problem wholly on STG domain for STGs containing multi-cycle signals and conditional behaviors. Rather than approach directly on state-diagram, our procedure does not incur the exponential complexity with respect to the signal numbers. The state coding problem considered here is the realizable statecoding which is a more relaxed requirement than the unique state coding. For STGs with multi-cycle signals, single-cycle transformation is proposed to transform the problem into ones with single-cycle signals. The experimental results have shown the complexity for most cases is near that for an STG/MG-S. For STGs with conditional behaviors, two novel operations, branch reversing and self-loop unfolding are proposed to significantly simplify the verification process. After transformation with these two operations, this verification can be performed by the techniques for STG/MGs. The proposed algorithmic approaches have been shown successful on over thirty examples from academic and industry.

\section{References}

[1] T. A. Chu, "Synthesis of Self-Timed Control Circuits from Graphical Specifications", PhD thesis, MIT, June, 1987

[2] T. H.-Y. Meng, Robert W. Brodersen, David G. Messerschimitt, "Automatic Synthesis of Asynchronous Circuits from High-Level Specification," IEEE Trans. on CAD., Pp. 1185-1205, No. 11, 1989.

[3] L. Lavagno and Cho W. Moon, "Private Communication".

[4] Peter Vanbekbergen, Francky Catthoor, Jef Van Meerbergen, Hugo DE Man, "Optimized Synthesis of Asynchronous Control Circuits from Graph-theoretic Specifications," Proceeding of the International Conference on Computer-Aided Design, pp. 184-187, 1990.

[5] K. J. Lin and C. S. Lin, "Automatic Synthesis of Asynchronous Circuits," Proceeding of 28th Design Automatic Conference, pp. 296-301, 1991.

[6] L. Lavagno, K. Keutzer, A. Sangiovanni Vincentelli, "Algorithms for Synthesis of Hazard-free Asynchronous Circuits," Proceeding of 28th Design Automatic Conference, pp. 302-308, 1991.

[7] Cho W. Moon, Paul R. Stephan and Robert K. Brayton, "Synthesis of Hazard-free Asynchronous Circuits from Graphical Specifications," Proceeding of the International Conference on Computer-Aided Design, pp. 322-325, 1991

[8] K. J. Lin and C. S. Lin, "A Realization Algorithm of of Asynchronous Circuits from STG," Proceeding of 1992 European Conference on Design Automation, pp. 322-326.

[9] J. L. Peterson, "Petri net theory and the modeling of system," prentice Hall, 1981.

[10] K. J. Lin, "Automatic Synthesis of Asynchronous Circuits," Ms. thesis, EE Department of National Taiwan University, 1990.

[11] K. J. Lin and C. S. Lin, "The verification of StateCoding in STGs," Technical report, EE Department of National Taiwan University, 1992.

\begin{tabular}{|c|c|c|c|c|c|}
\hline Circuits (a) & T & MC & MG & CB & VSC \\
\hline alloc-outbound-chu1.g & 29 & 2 & 2 & 1 & 1 \\
\hline alloc-outbound.g & 22 & 0 & 2 & 1 & 0 \\
\hline chu150.g & 14 & 1 & 1 & 0 & 1 \\
\hline chu172.g & 13 & 0 & 2 & 1 & 0 \\
\hline converta.g & 14 & 2 & 1 & 0 & 1 \\
\hline ebergen.g & 14 & 2 & 1 & 0 & 1 \\
\hline hazard.g & 10 & 1 & 1 & 0 & 1 \\
\hline pe-rcv-ifc.g & 50 & 4 & 6 & 3 & 2 \\
\hline rcv-setup.g & 12 & 0 & 3 & 2 & 0 \\
\hline sbuf-read-ctl.g & 19 & 0 & 2 & 1 & 0 \\
\hline sbuf-send-ctl.g & 24 & 2 & 2 & 1 & 1 \\
\hline sbuf-send-pkt2.8 & 25 & 1 & 4 & 5 & 1 \\
\hline stpl.g & 16 & 2 & 1 & 0 & 1 \\
\hline rpdft.g & 22 & 3 & 1 & 0 & 3 \\
\hline nowick.g & 16 & 2 & 1 & 0 & 1 \\
\hline wrdata.g & 12 & 1 & 1 & 0 & 1 \\
\hline wrdatab.g & 24 & 2 & 1 & 0 & 1 \\
\hline Lavagno.g & 16 & 3 & 1 & 0 & (b) \\
\hline pe-send-ifc.g & 52 & 8 & 5 & 6 & (c) \\
\hline
\end{tabular}

T: No. of transitions.

MC: Maximal No. of Multi-Cycle signals in MGs.

MG: No. of MG-component.

CB: Pairs of Conflict-Branches.

VSC: Iterations of Virtual Single-Cycle signals.

(a) Some STG/FCs are rewritten by merging apparent equivalent structures in conflict-branches.

(b) (c) The RSC verification for this STG/MG requires general single-cycle transformation on some multi-cycle signals.

Table 1. Experimental Results. 